\title{
EXPERIMENTAL STUDY OF SURFACE TEXTURE OT SHEET METAL WITH POLYESTER COATINGS
}

\author{
Andrei MAKSIMOV, Olga MAKSIMOVA \\ Cherepovets State University, Chair of Physics, Cherepovets, Russian Federation, \\ a v maximov@mail.ru
}

https://doi.org/10.37904/metal.2019.738

\begin{abstract}
Metal products with ttextured polymer coatings are distinguished by high strength, durability and esthetic qualities, which give to building structures (roof, etc.) a natural and noble appearance. At modern metallurgical enterprises, the correspondence of the color and texture of the polymer coating with the required parameters (color difference, gloss, etc.) is estimated often visually ("by eye"), without the use of magnifying devices. At present, the development of a methodology for comparison of optical properties of such coatings of metals with a standard is actual.

In this paper, the optical microscopy method was used to investigate the surface of metal-rolled samples with the textured polymer coatings of "steel silk" type. The dependences of the number of defects ("stars"), their parameters and the distribution over the surface (concentration of stars and the number of their rays) are analysized for the polyester coatings samples obtained under different thickness of the facial layer.
\end{abstract}

Keywords: Optical microscopy, metals with polymer coatings, color difference, gloss

\section{INTRODUCTION}

It is known, that many physical phenomena on smooth surfaces of solid bodies differ from phenomena on rough ones. For example, the light scattering on smooth surfaces is almost completely absent, and therefore, they are preferable, for example, in the manufacture of laser drives, which require accurate measurements and control of their surface roughness in order to ensure the quality and quantity of electrical components installed on the drive itself [1].

Surface roughness manifests itself as defects for many types of products in various industries. Therefore, the study and accurate measurement of the surface roughness is of practical importance, especially in the precision engineering and manufacturing, where strict requirements are imposed on the quality of the coating material and the miniaturization of product components.

However, in the last decade, there has been a need for new materials with additional design functions, etc., for example, in textured polymer coatings of sheet metal in metallurgy or products with special coatings in the textile, automotive and other industries. Due to the roughness of the surface, additional mechanical, optical, and other effects arise due to strong light scattering on the surfaces of such coatings [2], for example, high color variability of different samples, which leads to poor quality products. The development of promising technologies for the deposition of polymer coatings on sheet metal with a reduced variability is one of the most important tasks for production lines of such coatings. Currently, the rolled metal with a textured coating is characterized by high strength, durability and unique aesthetic qualities that give building structures (roof, etc.) a natural and noble appearance. However, at modern metallurgical enterprises, the correspondence between the color and texture of the polymer coating with the required parameters [3] (color variability, gloss, etc.) is estimated visually ("by eye") without using any devices. The use of photometric devices with directional receivers (detectors) of radiation is unsuccessful to measure the brightness, color properties and different shades due to strong light scattering on rough surfaces with complex relief (textured and other coatings). 
Therefore, at present, it is important to develop a method for comparing (converging) the optical properties of such a metal rolling with a standard.

The most well-known methods of non-destructive testing of surfaces with complex relief of metal coatings [5], alloys [6] and composites [7] are atomic force microscopy (AFM) and scanning probe microscopy (SPM) methods, which are used to study their microstructures (roughness, thickness, etc.) and mechanical characteristics. However, these and other similar methods are difficult to use under production conditions, as they require special expensive equipment, procedures for preparing samples and conditions for laboratories (the absence of vibrations, etc.). The purpose of this work is an experimental study of the surface texture of sheet metal with polymer coatings by conventional optical microscopy (OM) method with a resolution sufficient to analyze the dependence of surface texture details on the conditions of their formation.

\section{EXPERIMENTAL SAMPLES AND METHODS}

In this work, for an experimental study of the surface texture of metal-roll samples with polyester coatings of the type "steel silk" an optical microscope with a 56-fold magnification was used, The samples with a metal thickness of $0.5 \mathrm{~mm}$ and the facial layer with a RAL 3005 palette (wine-red, cherry ) were obtained at PAO Severstal (Cherepovets). The color of the palette of all samples was chosen RAL 3005, as it is widely used for facing materials in many types of construction. The resolution of the microscope was quite enough to analyze the texture of the coating. The peak temperatures of the facial and primer layers during the formation of the coatings were respectively equal to $230^{\circ}$ and $220^{\circ}$. The data of the four samples are given in Table 1. Scanning probe microscopy (SPM) was used to measure finer texture details..

Table 1 Samples of steel with polyester coating with RAL 3005 color

\begin{tabular}{|c|c|c|c|c|}
\hline \multirow{2}{*}{$\begin{array}{c}\text { Sample } \\
\text { number }\end{array}$} & \multicolumn{2}{|c|}{ The thickness of the coating layers $(\boldsymbol{\mu m})$} & \multirow{2}{*}{$\begin{array}{c}\text { The number of } \\
\text { rays stars }\end{array}$} & The Beam Width $(\boldsymbol{\mu m})$ \\
\cline { 2 - 3 } & Priming & Facial & $5-7$ & \\
\hline 1 & 7.28 & 16.07 & $10-12$ & 9.5 \\
\hline 2 & 6.12 & 15.76 & $10-14$ & 14.5 \\
\hline 3 & 5.43 & 14.02 & $12-14$ & 16.0 \\
\hline 4 & 8.13 & 11.30 & & 17.5 \\
\hline
\end{tabular}

The thickness of the layers: the primer (L1) and the front (L2) polymer coating was measured using the destruction of these facial layers by drilling out a crater passing through all layers of the coating to the metal substrate and its further analysis using the video microscope (Figure 1).

Figure 1 Image of the crater and the procedure for measuring the thickness of the coating

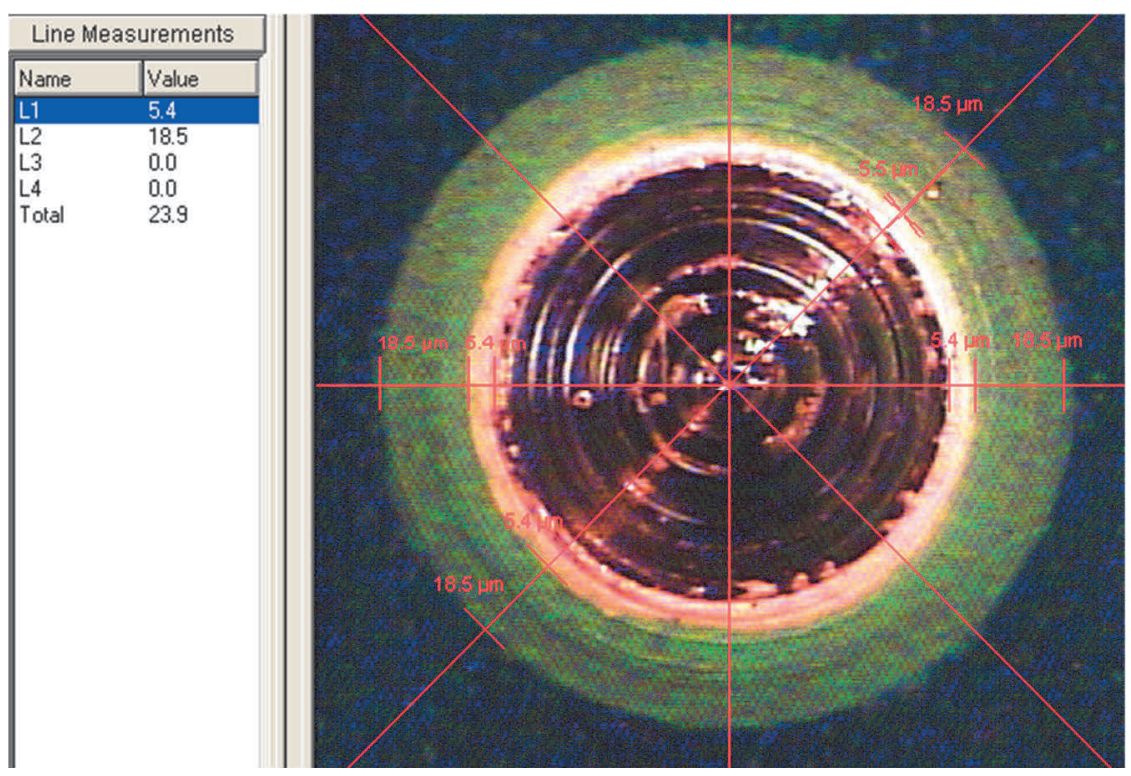




\section{RESULTS AND ITS DISCUSSION}

In the production of textured polymer sheet metal sheet coatings, special dopants are usually added to the paint material (PM) - granules, on which PM flocculate and further, the texture on the surface of the facial layer of coatings is formed. The most characteristic surface textures of the four samples of sheet metal with polyester coatings are shown in Figures 2-3. Analysis of the results of measurements of the thickness of coatings and micrographs showed that sample 1 with the greatest thickness of the facial layer has the greatest color diversity ("by eye"), and during the formation of its surface, the coatings spread and its further flotation happens on the granules with the formation of fairly small thickening centers - "stars" with a small number of "rays "(5-7 in total) leaving these centers (Figure 2 a).

a)

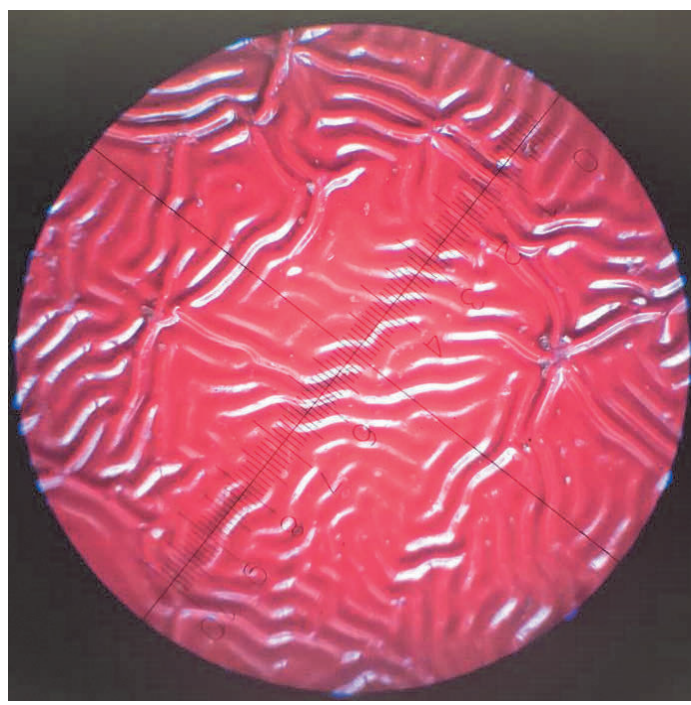

b)

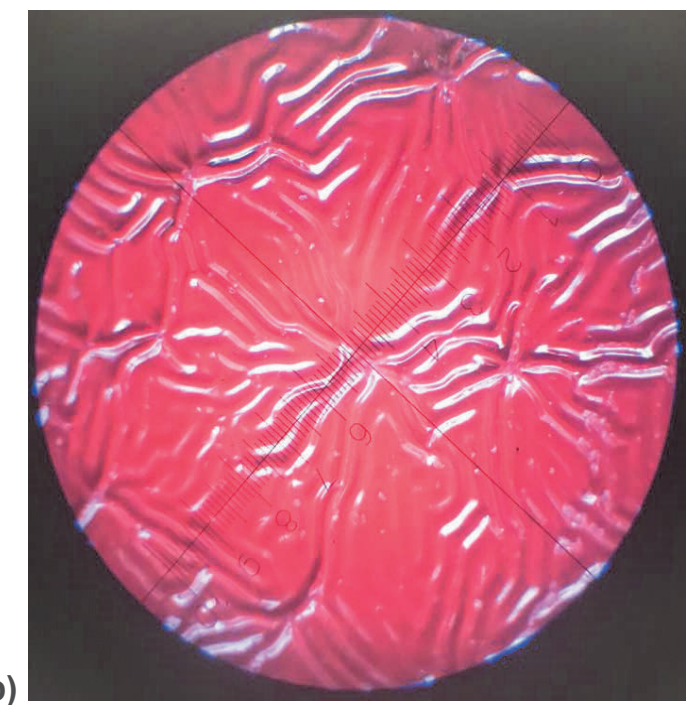

Figure 2 Micrographs of surfaces of the sheet metal samples 1 (a) and 2 (b) with polyester coating

The samples 2-4 with a smaller thickness of the facial layer have smaller color diversity, and clearly defined "stars" with 10 to 14 "rays" are visible on their surface (Figure $\mathbf{2}$ b and Figure $\mathbf{3}$ ). Thus, sample 4 with the minimum color diversity has the smallest thickness. However, in the production of textured polymer coatings, it does not make sense to decrease the thickness of this layer is less than 11.5 microns so that samples are obtained with a lower corrosion resistance.

a)

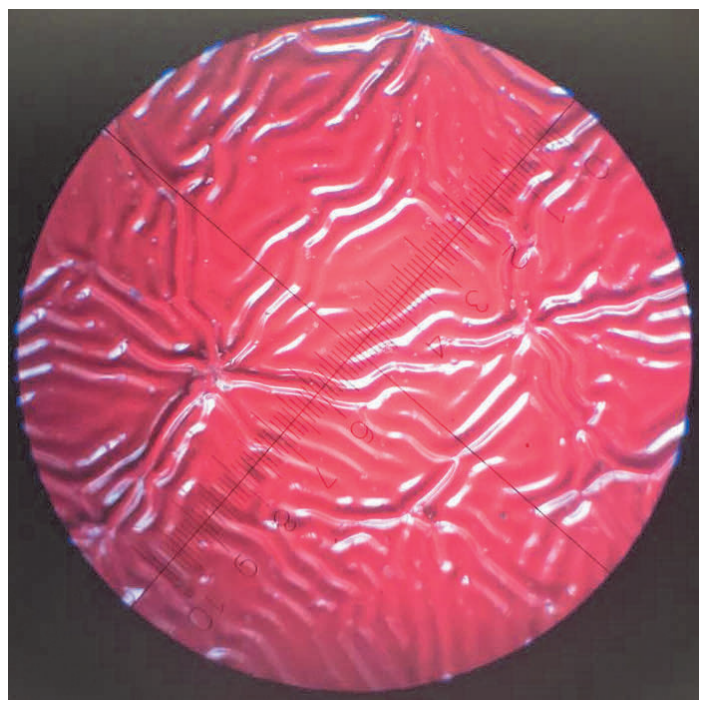

b)

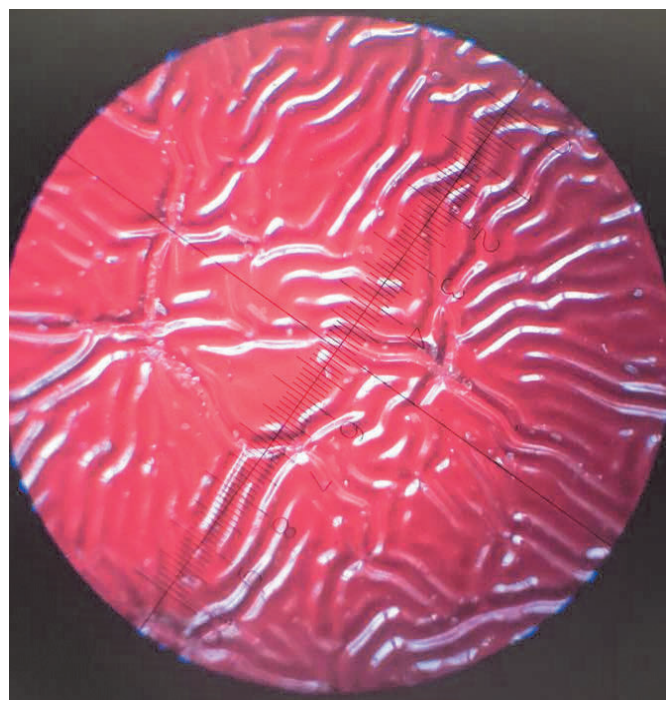

Figure 3 Micrographs of surfaces of the sheet metal samples $3(a)$ and $4(b)$ with polyester coating 
The width of the rays was measured the by scanning probe microscopy method (SPM) for the samples 1 and 4. It was equal to 9.5 and $17,5 \mu \mathrm{m}$, respectively (Figure 4).

a)

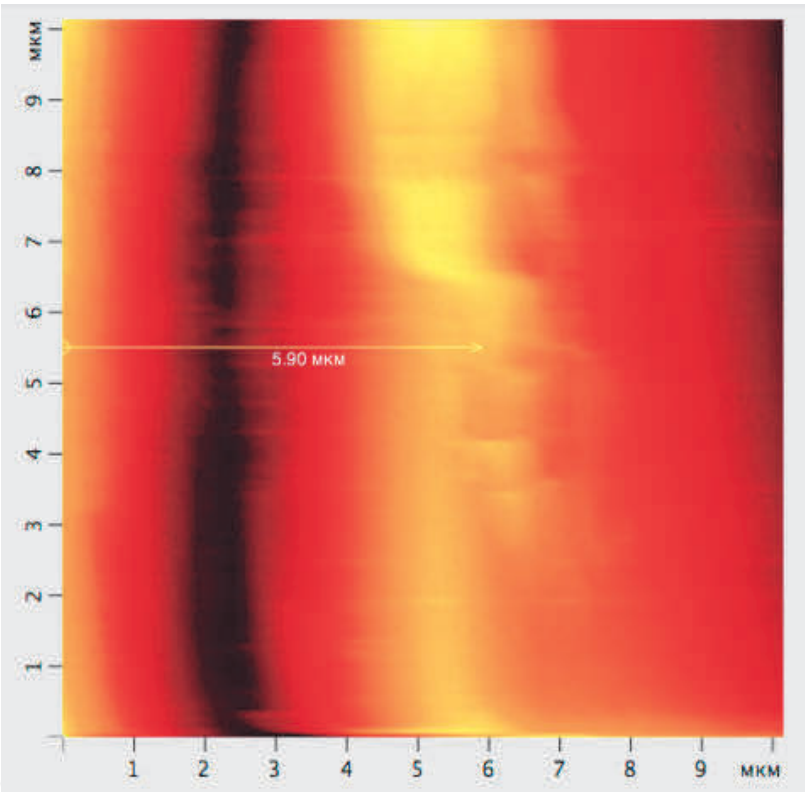

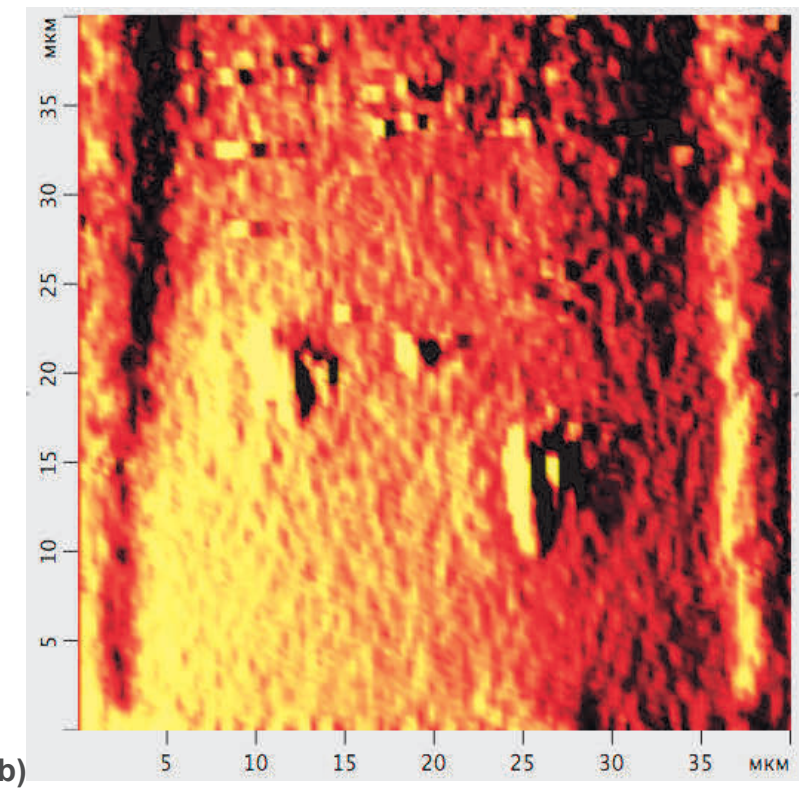

1 (a) and 3 (b) of sheet metal with polyester

The much larger width of the rays of the "stars" for the sample 4 as compared to the sample 1 leads to different light scattering and, accordingly, different surface differences of these samples.

\section{CONCLUSION}

Thus, the resolution of the optical microscopy method is quite sufficient for analyzing the surface texture of metal sheet with polymer coatings, and it can be used instead of the more labor-intensive SPM method, which is usually used for a more detailed analysis of the texture of coatings. The results obtained by the authors in this work have shown that at other equal conditions (furnace temperatures, etc.), the most important indicator in the formation of both textured and smooth polymer coatings is the thickness of the facial layer of the coating. These results are confirmed by the data obtained by means computer simulaton by the random walk method [7]. In diffusion limited cluster aggregation model used in the random walk method, the surface of the coating is represented as an array of the clusters of interconnected fractal objects.

The optical microscopy method can also be used to further develop the texture-color convergence technique for these and other textured polymer coatings. This technique is very relevant to improve the quality of sheet metal with polymer coatings. However, it should be noted that in this work, the effect of a batch of paint on the surface texture was not sufficiently studied: the authors simply did not have other samples. For clarifying studies of the results of this work and the development of the above convergence methodology, a reference standard with the Ral 3005 palette is required and also samples with other palettes for which the color variability appears more often (for example, Ral 3005 (yellow-zinc)).

\section{ACKNOWLEDGEMENTS}

The study was supported by the Russian Foundation for Basic Research, Project № 19-42-350001. 


\section{REFERENCES}

[1] SEMCHUK, Olexander, GRECHKO, Leonid, VODOPIANOV, Dmitriy, KUNITSKA, Leonid. Features of light scattering by surface fractal structures. TASK QUARTERLY. 2009. vol.13, no. 3, pp. 199-206.

[2] ZAMANI, Morteza, SHAFIEI, Farbod, FAZELI, Seyed Mahdi, DOWNER, Michael, JAFARI, Gholam Reza. Analytic height correlation function of rough surfaces derived from light scattering. Physical Review E. 2016. vol. 94, p. 042809.

[3] MAKSIMOV, Andrey, MAKSIMOVA, Ekaterina, EGOROV Vladislav. Modeling of light scattering by polymer coatings of rolled metal surface IOP Conf. Series: Journal of Physics: Conf. Series. 2017. vol. 936 012041, doi:10.1088/1742-6596/936/1/012041.2017

[4] GOST 34180-2017. Steel cold rolled and cold rolled hot-galvanized sheet with polymer coating, prepainted by the continuous coil-coating process. Specifications.

[5] Petrova, Tat'jana, Osipova, Nadegda Maksimov, Andrey, Panova, Marina, and Maksimova Olga. Using the SPM method to determine the convergence of rolled metal with textured polymer coatings, In Abstract Book of Internaional Conference "Scanning Probe Microscopy". Ekaterinburg: Ekaterinburg, Ural Federal University. 2017. pp.126-127.

[6] ABREUA C.M., ACUNAA, R., CABEZAA, M.M., CRISTÓBALA, J., MERINOA P., and VERDERAB, D. Microstructure and mechanical properties of $\mathrm{Al} / \mathrm{SiC}$ composite surface layer produced by friction stir processing. In Ciência\&Tecnologia dos Materiais, 2017. vol 1, iss. 1 pp.82-86.

[7] SADYRIN, E.V, MITRIN B.I,, KRENEV L.I. and AIZIKOVICH, S.M. Study of the mechanical and microgeometric characteristics of the TiN coating on a 40x steel substrate applied by ion plasma spraying.Vestnik Donskogo gosudarstvennogo universiteta. 2015. vol. 83, iss. pp. 37-45.

[8] MAKSIMOVA, Olga, BAIDGANOV Alexander, PETROVA Tatiana, MAKSIMOV, Andrei, EGOROV Vladislav. Simulation of the surface of metal sheet with textured polymer coating. In METAL 2019: 28rd International Conference on Metallurgy and Materials. Ostrava: TANGER, 2019 (in printed). 